\title{
Pipeline Embolization Device for Intracranial Aneurysms in a Large Chinese Cohort: Complication Risk Factor Analysis
}

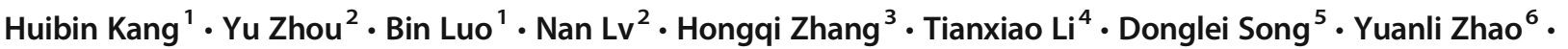 \\ Sheng Guan ${ }^{7}$. Aisha Maimaitili ${ }^{8} \cdot$ Yunyan Wang ${ }^{9} \cdot$ Wenfeng Feng $^{10} \cdot$ Yang Wang $^{11}$ - Jieqing Wan ${ }^{12} \cdot$ Guohua Mao $^{13}$. \\ Huaizhang Shi ${ }^{14} \cdot$ Xinjian Yang ${ }^{1} \cdot$ Jianmin Liu ${ }^{2}$
}

Accepted: 8 December 2020 / Published online: 14 January 2021

(C) The American Society for Experimental NeuroTherapeutics, Inc. 2021

\begin{abstract}
During intracranial aneurysm embolization with the Pipeline embolization device (PED), ischemic and hemorrhagic complications have been observed in cases among Western populations. The postmarket multicenter registry study on the embolization of intracranial aneurysms with the PED in China, i.e., the PLUS study, was performed to assess real-world predictors of complications and functional outcomes in patients treated with the PED in a Chinese population. All patients with intracranial aneurysms who underwent embolization using the PED between November 2014 and October 2019 across 14 centers in China were included. The study endpoints included preoperative and early postoperative $(<30$ days) functional outcomes (modified Rankin scale [mRS] scores) and complications related to PED treatment at early postoperative and follow-up time points (3-36 months). Multivariate analysis was performed to identify risk factors for complications. A total of 1171 consecutive patients (mean age, 53.9 \pm 11.4 ; female, 69.6\% [813/1171]) with 1322 aneurysms were included in the study. Hypertension, basilar artery aneurysms, and successful deployment after adjustment or unsuccessful device deployment were found to be independent predictors of ischemic stroke, while the use of the Flex PED and incomplete occlusion immediately after treatment were protective factors. An aneurysm size $>10 \mathrm{~mm}$, distal anterior circulation aneurysms, and adjunctive coiling were found to be independent predictors of delayed aneurysm rupture, distal intraparenchymal hemorrhage, and neurological compression symptoms, respectively. The rate of PED-related complications in the PLUS study was similar to that in Western populations. The PLUS study identified successful deployment after adjustment or unsuccessful device deployment and the degree of immediate postoperative occlusion as novel independent predictors of PEDrelated ischemic stroke in a Chinese population. ClinicalTrial.gov Identifier: NCT03831672
\end{abstract}

Key Words Complications · intracranial aneurysms · China · ischemic $\cdot$ multi-center · endovascular treatment · Pipeline embolization device

Huibin Kang and Yu Zhou share first authorship.

Xinjian Yang

yangxinjian@voiceoftiantan.org

$\triangle$ Jianmin Liu

chstroke@163.com

1 Department of Interventional Neuroradiology, Beijing Neurosurgical Institute and Beijing Tiantan Hospital, Capital Medical University, No. 119 South Fourth Ring West Road, Fengtai District, Beijing 100050, China

2 Department of Neurosurgery, Changhai Hospital, Naval Medical University, 168 Changhai Rd, Shanghai 200433, China

3 Xuanwu Hospital, Capital Medical University, Beijing, China

4 Zhengzhou University People's Hospital, Zhengzhou, China

5 Shanghai Donglei Brain Hospital, Shanghai, China
6 Peking University International Hospital, Beijing, China

7 First Affiliated Hospital of Zhengzhou University, Zhengzhou, China

8 First Affiliated Hospital of Xinjiang Medical University, Uruqi, China

9 Qilu Hospital of Shandong University, Jinan, China

10 Nanfang Hospital, Southern Medical University, Guangzhou, China

11 First Affiliated Hospital of Nanchang University, Nanchang, China

12 Renji Hospital, School of Medicine, Shanghai Jiao Tong University, Shanghai, China

13 Second Affiliated Hospital of Nanchang University, Nanchang, China

14 First Affiliated Hospital of Harbin Medical University, Harbin, China 


\section{Abbreviations}

PED Pipeline embolization device

PLUS Postmarket multicenter retrospective research on embolization of intracranial aneurysms with Pipeline embolization devices in China

DAR Delayed aneurysm rupture

DICH Distal intraparenchymal hemorrhage

TIA Transient ischemic attack

SAH Subarachnoid hemorrhage

DSA Digital subtraction angiography

EDC Electronic data capture

mRS Modified Rankin scale

OR Odds ratio

\section{Introduction}

The Pipeline embolization device (PED, Covidien/Medtronic) is widely used to treat patients with intracranial aneurysms and has demonstrated high rates of occlusion in large cohort studies [1-4]. However, the safety of the PED remains a concern, with a non-negligible risk of postoperative hemorrhage $(2.4 \%)$ and ischemic stroke (4.7\%) [5]. These complications mostly occur in the early postoperative period and mainly involve delayed aneurysm rupture (DAR) and distal intraparenchymal hemorrhage (DIPH), as reported among Western populations $[3,6,7]$. Importantly, Chinese populations differ from Western populations in the prevalence of comorbidities such as intracerebral hemorrhage (ICH) and intracranial atherosclerosis, which may influence the risk of ischemic stroke [8,9]. Large, multicenter studies describing the incidence and risk factors for complications related to PED use in Chinese populations are lacking. Thus, the purpose of this postmarket multicenter registry study on the embolization of intracranial aneurysms with the PED in China, i.e., the PLUS study, was to assess the real-world predictors of complications and functional outcomes in patients with intracranial aneurysms treated with the PED in a Chinese population.

\section{Methods}

\section{Ethics Approval}

The study protocol was reviewed and approved by the ethics committee of Beijing Tiantan Hospital, and the approval number given by the ethical board was KY 2018-098-02. All patients provided written informed consent.

\section{Study Design and Participants}

All patients with intracranial aneurysms who underwent embolization using the PED between November 2014 and
October 2019 in 14 centers in China were included. This study included patients with ruptured and unruptured aneurysms, aneurysms of different morphologies, including saccular, dissecting, fusiform, and blister aneurysms, and aneurysms located at different intracranial sites.

Aneurysms were classified based on the maximum diameter (small $[<10 \mathrm{~mm}]$, large $[10-25 \mathrm{~mm}]$, and giant $[\geq$ $25 \mathrm{~mm}]$ ), treatment modality (PED alone and PED plus coiling), and location (anterior or posterior circulation). The anterior circulation was divided into the proximal and distal anterior circulation. The proximal anterior circulation was defined as arteries from the beginning of the internal carotid artery to the bifurcation of the internal carotid artery, including the ophthalmic artery, arterial segment in the cavernous sinus, posterior communicating artery, superior hypophyseal artery, and posterior carotid wall. The distal anterior circulation was defined as the arteries branching from the internal carotid artery, including the anterior and middle cerebral arteries. Patient demographics, aneurysm characteristics, the antiplatelet regimen after platelet function testing, and procedural details were recorded.

\section{Procedural Details}

Embolization procedures involved the use of the Classic or Flex PED. The PED was delivered and deployed through a Marksman microcatheter (Medtronic, Irvine, California). Additional techniques, including the use of adjunctive coils, overlapping PEDs, and balloon angioplasty, were applied according to the operator's preference and experience. Because the study was conducted retrospectively, the protocol did not specify the coiling methodology, and the decision to use coils with the PED was left to the discretion of the treating physician. Patients were administered an antiplatelet regimen that included a combination of aspirin (100-300 mg daily) and clopidogrel (75 mg daily); however, patients who were identified as clopidogrel non-responders were administered aspirin (100 mg daily) and ticagrelor ( $90 \mathrm{mg}$ twice daily). The dose of aspirin/clopidogrel was adjusted preoperatively after platelet function testing. Platelet function testing was performed in the same manner at all sites.

\section{Study Outcomes}

The study outcomes included complications related to PED treatment, all of which were adjudicated by a central review committee. Complications included the following: access site complications; major ischemic stroke (defined as a change in the National Institutes of Health Stroke Scale [NIHSS] score of $>4$ lasting more than 7 days); minor neurological stroke (defined as a change in the NIHSS score of $\leq 4$ lasting fewer than 7 days with corroborative imaging) and transient ischemic attack (TIA, defined as a transient neurological deficit 
without corroborative imaging [10]) analyzed as a combined outcome; DAR; DIPH; neurological compression symptoms (emergent or accentuated postoperatively); asymptomatic $\mathrm{ICH}$; parent artery stenosis; stent migration; mortality; and preoperative and early postoperative ( $<30$ days) functional outcomes assessed using the modified Rankin scale (mRS). Neurological compression symptoms were evaluated by experienced neurologists and were defined as cranial neuropathy or brainstem symptoms caused by aneurysm compression.

Device deployment to the target site was considered unsuccessful when PED opening failed or when the PED was deployed inside the aneurysm. Successful device deployment to the target site after adjustment was defined when the PED was released successfully after technical adjustments. Device deployment to the target site was considered successful when the PED was released successfully at the target location without technical adjustments.

\section{Patient Follow-up}

Early postoperative follow-up was performed at $<30$ days. Follow-up patient imaging was performed at 3, 6, 12, 24, and 36 months. Patients who underwent follow-up participated in at least one of these follow-up examinations. The data from the last follow-up examination for each patient were used for calculation. Aneurysm occlusion was determined using digital subtraction angiography (DSA). New or recurrent subarachnoid hemorrhage $(\mathrm{SAH})$ or intraparenchymal hemorrhage (IPH) was evaluated on head computed tomography (CT) in the case of clinical deterioration and adjudicated if necessary. Clinical follow-up was performed for all patients, regardless of imaging availability, and was conducted by telephone or by email.

A central review committee consisted of 3 members, including a neurointerventionist, a medical imaging doctor, and a neurosurgeon, who reviewed all imaging data and endpoint events. Each evaluation was performed independently by two members; in cases of dispute, the team reached a consensus after discussion. Electronic data capture (EDC) was used for data collection.

\section{Statistical Analysis}

Data are presented as the mean and range for continuous variables and as frequencies for categorical variables. The analysis was carried out using unpaired $t$ tests (continuous variables), chi-square tests (categorical variables), and Fisher's exact tests. Univariate analysis was used to test covariates predictive of the following dependent variables: ischemic stroke (in the early postoperative period, the follow-up period, and throughout the study duration), DAR, DIPH, neurological compression symptoms, neurological mortality, and poor functional outcomes in the early postoperative period.
Interactions and confounding variables were assessed through stratification and relevant expansion covariates. Factors predictive on univariate analysis were entered into a multivariate logistic regression analysis [11]. $P$ values of $<0.05$ were considered statistically significant. Statistical analysis was carried out with SPSS version 25 (IBM Corp., Armonk, NY, USA).

\section{Results}

\section{Baseline Characteristics}

Demographic and baseline characteristics are presented in Table 1. A total of 1171 patients with 1322 aneurysms were treated using the PED at 14 centers in China. The mean age was $53.9 \pm 11.4$ years, and $69.4 \%(813 / 1171)$ of patients were female. Comorbidities included hypertension (33.9\% [397/ 1171]), diabetes (5.4\% [63/1171]), hyperlipidemia (3.6\% [42/ 1171]), cerebral infarction (4.6\% [54/1171]), cardiac disease (5.0\% [58/1171]), and cerebral atherosclerosis (14.9\% [174/ $1171])$. Aneurysms were symptomatic in $60.1 \%$ (704/1171) of patients and incidental in $36.3 \%$ (425/1171) of patients, and $3.2 \%$ (42/1171) of aneurysms involved SAH (SAH ictus to aneurysm treatment: within $72 \mathrm{~h}$ in 6 cases, within 4-7 days in 18 cases, within 8-15 days in 8 cases, and within 15-30 days in 10 cases).

\section{Aneurysm Characteristics}

Aneurysm characteristics are presented in Table 2. The mean aneurysm size, neck size, and parent artery diameter was $12.79 \pm 8.75 \mathrm{~mm}, 6.21 \pm 3.92 \mathrm{~mm}$, and $3.88 \pm 0.82 \mathrm{~mm}$, respectively. The morphology of aneurysms was saccular in $83.1 \%$ (1099/1322), fusiform in 8.2\% (109/1322), dissecting in $6.3 \%(83 / 1322)$, and blister in $2.3 \%(31 / 1322)$ of cases. The majority of occlusions were located in the anterior circulation (proximal, 83.6\% [1105/1322]; distal, 3.6\% [48/1322]), while $12.8 \%(169 / 1322)$ were located in the posterior circulation, including $2.2 \%(29 / 1322)$ in the basilar artery and $10.6 \%$ $(140 / 1322)$ in the vertebral artery and other vessels in the posterior circulation.

\section{Procedural Details and Angiographic and Clinical Outcomes}

Treatment details and angiographic and clinical outcomes are presented in Table 3. The Classic and Flex PEDs were used in similar proportions $(45.2 \%$ [596/1322] vs. 54.8\% [723/1322], respectively). The diameter and length of the device was 4.07 $\pm 0.53 \mathrm{~mm}$ and $25.37 \pm 5.76 \mathrm{~mm}$, respectively.

At the last follow-up for each patient, the total occlusion rate was $81.4 \%(787 / 967), 91.6 \%(1211 / 1322)$ of parent arteries were patent, $7.0 \%(93 / 1322)$ of parent arteries were stenotic, and $1.4 \%$ (18/1322) of parent arteries were occluded. A 
Table 1 Baseline characteristics of 1171 patients treated with the PED

\begin{tabular}{ll}
\hline Characteristic & $\begin{array}{l}\text { Frequency } \\
(N=1171)\end{array}$ \\
\hline Total number of aneurysms treated with the PED & 1322 \\
Patients with multiple aneurysms & $260(22.2 \%)$ \\
Female & $813(69.4 \%)$ \\
Age & $53.9 \pm 11.4$ \\
Family history of aneurysm & $18(1.5 \%)$ \\
Comorbidities & \\
Hypertension & $397(33.9 \%)$ \\
Diabetes & $63(5.4 \%)$ \\
Hyperlipidemia & $42(3.6 \%)$ \\
Cerebral infarction & $54(4.6 \%)$ \\
Cardiac disease & $58(5.0 \%)$ \\
Cerebral atherosclerosis & $174(14.9 \%)$ \\
Alcohol abuse & \\
Never & $1027(87.7 \%)$ \\
Previous & $118(10.1 \%)$ \\
Current & $26(2.2 \%)$ \\
Smoking & \\
Never & $863(73.7 \%)$ \\
Previous & $91(7.8 \%)$ \\
Current & $217(18.5 \%)$ \\
Presentation & \\
Sycidental & $425(36.3 \%)$ \\
Current SAH & $704(60.1 \%)$ \\
& $42(3.2 \%)$ \\
\hline
\end{tabular}

Data are shown as $n(\%)$ or the mean $\pm \mathrm{SD}$

total of $93.9 \%$ (1241/1322) of PEDs were successfully deployed to the target site, and the rate of complete occlusion immediately after the operation was $14.6 \%$.

The mRS score was $0-2$ and 3-6 before PED treatment in $1060(90.5 \%)$ and $111(9.5 \%)$ cases, respectively. The mRS score was $0-2$ and 3-6 in the early postoperative in 1094 (93.4\%) and 77 (6.6\%) cases, respectively. The mRS score was $0-2$ and 3-6 at follow-up in 1111 (94.9\%) and 60 $(5.1 \%)$ cases, respectively. The mean imaging follow-up time was $8.96 \pm 7.50$ months (median, 6 months; range, 336 months). In, $26.6 \%(313 / 1171)$ of cases, the patient did not reach the follow-up time or was lost to imaging followup; no patients were lost to clinical follow-up.

\section{Morbidity, Mortality, Complications, and Functional Outcomes}

Table 4 presents the study outcomes. Major ischemic stroke occurred in 3.8\% (44/1171) of patients in the early postoperative period and $0.6 \%$ (7/1171) in the follow-up period. Minor stroke and/or TIA occurred in $2.6 \%$ (30/1171) of patients in the
Table 2 Aneurysm characteristics

\begin{tabular}{ll}
\hline Characteristic & Frequency $(N=1322)$ \\
\hline Aneurysm size (maximum aneurysm length) & $12.79 \pm 8.75$ \\
$<10 \mathrm{~mm}$ & $630(47.7 \%)$ \\
$10-25 \mathrm{~mm}$ & $555(42.0 \%)$ \\
$>25 \mathrm{~mm}$ & $137(10.4 \%)$ \\
Average neck size & $6.21 \pm 3.92$ \\
Average parent artery diameter & $3.88 \pm 0.82$ \\
Aneurysm form & \\
Saccular & $1099(83.1 \%)$ \\
Fusiform & $109(8.2 \%)$ \\
Dissecting & $83(6.3 \%)$ \\
Blister & $31(2.3 \%)$ \\
Location & \\
Anterior circulation & \\
$\quad$ Anterior circulation proximal & $1105(83.6 \%)$ \\
Anterior circulation distal & $48(3.6 \%)$ \\
Posterior circulation & $169(12.8 \%)$ \\
Basilar & $29(2.2 \%)$ \\
Vertebral artery and other vessels in the & $140(10.6 \%)$ \\
posterior circulation* & \\
\hline
\end{tabular}

Data are shown as $n(\%)$ or the mean $\pm \mathrm{SD}$

*Other vessels in the posterior circulation include the posterior cerebral artery and posterior inferior cerebellar artery

early postoperative period and $0.3 \%(4 / 1171)$ in the follow-up period. DAR occurred in $2.0 \%$ (23/1171) of patients in the early postoperative period and $0.1 \%(1 / 1171)$ in the follow-up period. The rate of DIPH was $2.0 \%$ (23/1171) in the early postoperative period and $0 \%$ during the follow-up period. Access site complications occurred in 3.5\% (41/1171) of patients using femoral access. Stent migration in the early postoperative period occurred in $0.8 \%$ (9/1171) of cases versus $0.0 \%$ at the follow-up. Cranial neuropathy or brainstem symptoms caused by aneurysm compression occurred in 3.8\% (44/1171) of patients in the early postoperative period and $0.6 \%(7 / 1171)$ in the follow-up period. The mortality rate was $1.3 \%(15 / 1171)$ in the early postoperative period and $0.2 \%(2 / 1171)$ in the follow-up period. Poor functional outcomes were observed in $6.6 \%(77 / 1171)$ and $5.1 \%(60 / 1171)$ of patients in the early postoperative and follow-up periods, respectively.

\section{Predictors of Stroke}

The results of multivariate analysis for predictors of ischemic stroke, TIA, and minor stroke in the early postoperative period, follow-up period, and throughout the study duration are presented in Supplementary Table 1. In the early postoperative period, hypertension ( $\mathrm{OR}=1.743, P=0.017)$, basilar artery aneurysm location $(\mathrm{OR}=3.705, P=0.012)$, and 
Table 3 Treatment details and angiography follow-up outcomes of 1322 aneurysms

\begin{tabular}{|c|c|}
\hline Procedural characteristics & $\begin{array}{l}\text { Frequency ( } N=1322 \\
\text { aneurysms) }\end{array}$ \\
\hline \multicolumn{2}{|l|}{ PED type } \\
\hline Classic PED & $596(45.1 \%)$ \\
\hline Flex PED & $723(54.7 \%)$ \\
\hline \multicolumn{2}{|l|}{ PED size } \\
\hline Diameter (mm) & $4.07 \pm 0.53$ \\
\hline Length (mm) & $25.37 \pm 5.76$ \\
\hline PED + coiling & $637(48.2 \%)$ \\
\hline Loose packing & $504(38.1 \%)$ \\
\hline Dense packing & $133(10.1 \%)$ \\
\hline Balloon angioplasty & $31(2.3 \%)$ \\
\hline Multiple PEDs used & $75(5.7 \%)$ \\
\hline Single PED used & $178(13.5 \%)$ \\
\hline \multicolumn{2}{|l|}{ Blood thinners } \\
\hline PrePED aspirin/clopidogrel $>3$ days & 915/1171 (78.1\%) \\
\hline PostPED aspirin/clopidogrel $>6$ months & $676 / 1171(57.7 \%)$ \\
\hline Previous treatment & $33 / 1171(2.8 \%)$ \\
\hline Coiling & $11 / 33(33.3 \%)$ \\
\hline Stent-assisted coiling & $22 / 33(66.7 \%)$ \\
\hline Clipping & $2 / 33(6.1 \%)$ \\
\hline \multicolumn{2}{|l|}{ Angiographic outcomes } \\
\hline Number of covered collateral arteries & $927(70.1 \%)$ \\
\hline \multicolumn{2}{|l|}{ Status of covered collateral artery } \\
\hline Patent & $627(47.4 \%)$ \\
\hline Stenotic & $54(4.1 \%)$ \\
\hline Occluded & $26(2.0 \%)$ \\
\hline $100 \%$ aneurysm occlusion at last follow-up* & $787(81.4 \%)$ \\
\hline \multicolumn{2}{|l|}{ Parent artery status } \\
\hline Patent & $1211(91.6 \%)$ \\
\hline Stenotic & $93(7.0 \%)$ \\
\hline Occluded & $18(1.4 \%)$ \\
\hline \multicolumn{2}{|l|}{ Device deployment to the target $\operatorname{site}^{\dagger}$} \\
\hline Successful & $1241(93.9 \%)$ \\
\hline Successful after stent adjustment & $68(5.1 \%)$ \\
\hline Unsuccessful & $10(0.8 \%)$ \\
\hline \multicolumn{2}{|c|}{ Satisfactory occlusion immediately after PED treatment } \\
\hline Complete occlusion & $193(14.6 \%)$ \\
\hline Incomplete occlusion & $1129(85.4 \%)$ \\
\hline \multicolumn{2}{|l|}{ Clinical outcomes } \\
\hline \multicolumn{2}{|l|}{ mRS score } \\
\hline \multicolumn{2}{|l|}{ PrePED mRS score } \\
\hline $0-2$ & $1060(90.5 \%)$ \\
\hline $3-6$ & $111(9.5 \%)$ \\
\hline \multicolumn{2}{|l|}{ PostPED mRS score (<30 days) } \\
\hline $0-2$ & $1094(93.4 \%)$ \\
\hline $3-6$ & $77(6.6 \%)$ \\
\hline \multicolumn{2}{|l|}{ mRS score at follow-up } \\
\hline $0-2$ & $1111(94.9 \%)$ \\
\hline $3-6$ & $60(5.1 \%)$ \\
\hline Mean imaging follow-up time (months) & $8.96 \pm 7.50$ \\
\hline \multicolumn{2}{|l|}{ Imaging follow-up distribution (no. of patients) } \\
\hline 3 months & $250(18.9 \%)$ \\
\hline 6 months & $629(47.6 \%)$ \\
\hline 12 months & $211(16.0 \%)$ \\
\hline 24 months & $73(5.5 \%)$ \\
\hline 36 months & $44(3.3 \%)$ \\
\hline Imaging loss to follow-up & $313(23.7 \%)$ \\
\hline Clinical loss to follow-up & $0(0.0 \%)$ \\
\hline
\end{tabular}

Data are shown as $\mathrm{n}(\%), n / N(\%)$, or the mean $\pm \mathrm{SD}$

*A total of 858 patients with 967 aneurysms underwent angiography follow-up

$\dagger$ The total number of PEDs used was 1319

* There was more than one follow-up visit for the same patient successful device deployment after adjustment or unsuccessful device deployment $(\mathrm{OR}=2.833, P<0.001)$ were independent predictors of ischemic stroke, while Flex PED use (OR = $0.458, P=0.001)$ and incomplete occlusion immediately after the operation $(\mathrm{OR}=0.546, P=0.029)$ were protective factors for ischemic stroke.

In the follow-up period, predictors of ischemic stroke were a history of cerebral infarction (OR $=7.024, P=0.006)$, successful device deployment after adjustment or unsuccessful deployment to the target site $(\mathrm{OR}=6.944, P=0.007)$, and distal anterior circulation aneurysm location $(\mathrm{OR}=6.950$, $P=0.022)$. Hypertension $(\mathrm{OR}=1.793, P=0.009)$, basilar artery aneurysm location $(\mathrm{OR}=4.384, P=0.002)$, and successful deployment with adjustment or unsuccessful device deployment to the target site $(\mathrm{OR}=3.968, P<0.0001)$ were independent predictors of ischemic stroke, while Flex PED use (OR $=0.464, P=0.001)$ and incomplete occlusion immediately after the operation (OR $=0.524, P=0.015)$ were protective factors for ischemic stroke.

\section{Predictors of DAR in the Early Postoperative Period}

On multivariate analysis, a large aneurysm size, especially sizes $>10 \mathrm{~mm}$, was an independent predictor of DAR in the early postoperative period $(\mathrm{OR}=5.446, P=0.008)$ (Supplementary Table 2).

\section{Predictors of DIPH in the Early Postoperative Period}

On multivariate analysis, a distal anterior circulation aneurysm location was an independent predictor of DIPH $(\mathrm{OR}=$ 5.129, $P=0.005$ ) (Supplementary Table 3).

\section{Predictors of Neurological Compression Symptoms}

On multivariate analysis, adjunctive coiling was an independent predictor of neurological compression symptoms $(\mathrm{OR}=$ 3.105, $P<0.0001)$ (Supplementary Table 4).

\section{Predictors of Mortality}

On univariate and multivariate analyses, an aneurysm size $>10 \mathrm{~mm}(\mathrm{OR}=4.914, P=0.013)$ and a basilar artery aneurysm location $(\mathrm{OR}=9.582, P=0.001)$ were independent predictors of morbidity (Supplementary Table 5).

\section{Predictors of Poor Functional Outcomes}

On multivariate analysis, independent predictors of poor functional outcomes included a large aneurysm size $(\mathrm{OR}=1.789$, $P=0.020)$ and adjunctive coiling $(\mathrm{OR}=2.026, P=0.005)$ (Supplementary Table 6). 
Table 4 Morbidity, mortality, complications, and functional outcomes in the early postoperative period and followup period

\begin{tabular}{lll}
\hline Variable & $\begin{array}{l}\text { Early postoperative } \\
\text { period }(<30 \text { days })(N=1171)\end{array}$ & $\begin{array}{l}\text { Postoperative follow-up } \\
\text { period }(3-36 \text { months })(N=1171)\end{array}$ \\
\hline Major ischemic stroke & $44(3.8 \%)$ & $7(0.6 \%)$ \\
TIA/minor stroke & $30(2.6 \%)$ & $4(0.3 \%)$ \\
DAR & $23(2.0 \%)$ & $1(0.1 \%)$ \\
DIPH & $23(2.0 \%)$ & $0(0 \%)$ \\
Stent migration & $9(0.8 \%)$ & $0(0 \%)$ \\
Access site complications & $41(3.5 \%)$ & $0(0 \%)$ \\
Neurological compressive symptoms & $44(3.8 \%)$ & $7(0.6 \%)$ \\
Mortality & $15(1.3 \%)$ & $2(0.2 \%)$ \\
Poor functional outcome, mRS score 3-6 & $77(6.6 \%)$ & $60(5.1 \%)$ \\
\hline
\end{tabular}

Data are shown as $n(\%)$

\section{Discussion}

The PLUS study demonstrated low rates of complications and mortality related to PED use in the treatment of patients with intracranial aneurysms in China, and these rates are comparable to those in studies using the PED in Western populations $[5,12]$. Importantly, this study identified novel risk factors for ischemic stroke (i.e., successful device deployment after adjustment or unsuccessful deployment to the target site and degree of postoperative occlusion immediately after the operation) and showed that these risk factors vary between the early postoperative period and the longer-term follow-up period.

The cohort in the PLUS study was comparable in size to that in large Western studies. In a pooled analysis of three Western studies-IntrePED (International Retrospective Study of the Pipeline Embolization Device), PUFS (Pipeline for Uncoilable or Failed Aneurysms Study), and ASPIRe (Aneurysm Study of Pipeline in an Observational Registry)-Kallmes et al. reported an average age of 57.4 years, a female proportion of $81.3 \%$, and an average aneurysm size of $12.0 \mathrm{~mm}$, with $85.3 \%$ of the aneurysms located in the proximal anterior circulation [12]. The PLUS cohort was similar in terms of the average size and percentage of proximal anterior circulation aneurysms; however, it included a younger population with a greater proportion of males. Additionally, the percentage $(48.3 \%)$ of patients treated with the PED combined with coiling was higher than the $6.9 \%$ of aneurysms treated as such by Sweid et al. in a retrospective cohort of 598 aneurysms [13].

\section{Ischemic Stroke Complications}

The rate of ischemic stroke complications in the PLUS study was comparable to the major ipsilateral ischemic stroke rate of $3.7 \%$ reported for a pooled cohort by Kallmes et al. [12]. In the IntrePED study, the majority of strokes occurred within 30 days of treatment $(26 / 793,3.3 \%)$, with the highest rate in patients with posterior circulation aneurysms $(7.3 \%, 4 / 55)$ [5]. Similarly, a systematic review and meta-analysis of 29 studies including 1451 patients with 1654 aneurysms found that patients with anterior circulation aneurysms were at a lower risk of ischemic stroke (OR $=0.15, P<0.0001)$, particularly of perforator infarction [14]. However, in the PLUS study, the majority of major ischemic strokes occurred within the early postoperative period; basilar aneurysms were an independent risk predictor for ischemic stroke in the early postoperative period, and distal anterior circulation aneurysms carried a higher risk of ischemic stroke in the follow-up period.

Other reported predictors of major complications include Classic PED use as opposed to Flex PED use (OR $=3.7, P=$ $0.008)$, in situ thrombosis $(\mathrm{OR}=4.3, P=0.006)$, and device deployment in the anterior cerebral artery or middle cerebral artery, as opposed to the internal carotid artery $(\mathrm{OR}=3.5, P=$ 0.034 ) [10]. In the DIVERSION prospective cohort study in France, a high baseline blood pressure was associated with the occurrence of a neurological deficit [15]. Our data show that hypertension was an independent risk predictor for ischemic stroke in the early postoperative period, a history of cerebral infarction was an independent risk predictor for ischemic stroke in the follow-up period, and successful deployment with adjustment or unsuccessful device deployment to the target site was an independent risk predictor for ischemic stroke throughout the study duration, while Flex PED use was associated with a reduced incidence of ischemic stroke in the early postoperative period and throughout the duration of the study.

Our study also identified incomplete occlusion compared with complete occlusion immediately after the operation as a factor associated with a reduced incidence of ischemic stroke in the early postoperative period, which has not been previously reported. The higher rate of combined coiling in Chinese patients helped us to identify this novel independent predictor, in that the degree of occlusion immediately postoperatively was related to ischemic stroke in the Chinese population. The exact mechanism for these results is still unknown. 
A reasonable explanation is that the complete embolization of aneurysms requires a longer operation and more operative procedures, which may increase the ischemic stroke risk. The PLUS study suggests that successful deployment with adjustment or unsuccessful device deployment plays a role in the occurrence of ischemic complications. This may be explained by the endothelial damage resulting from repeated adjustment of the stent(s) after failure of the PED to open, leading to ischemia.

\section{DAR and DIPH}

Rouchaud et al. reported that approximately $80 \%$ of DARs occurred within 30 days of the procedure, $45 \%$ of which involved giant aneurysms [16]. In a retrospective study, the frequency of DAR was $1.2 \%, 88 \%$ of aneurysms re-ruptured during the first month, and a previous $\mathrm{SAH}$, a large aneurysm size, and a posterior circulation aneurysm location were predictors of DAR [13]. Comparatively, the PLUS cohort showed a slightly higher rate of DAR, with $95 \%$ of DARs occurring in the early postoperative period, and a large aneurysm size, especially sizes $>10 \mathrm{~mm}$, was an independent predictor of DAR in the early postoperative period. Our results are similar to the overall postoperative rupture rate of $4 \%$, early rupture rate of $3 \%$, and significantly higher rupture rate in large and giant aneurysms reported in the meta-analysis by Brinjikji et al. [14]. Computational fluid dynamic studies have demonstrated that an increase in intra-aneurysmal pressure may lead to rupture [17]. Although we found no association between the use of antiplatelet drugs and the risk of hemorrhagic complications in our study, DIPH has also been thought to be related to antiplatelet regimens, according to previous studies $[7,13]$. One may speculate that the increased metal surface area to which the platelets are exposed may result in activation through increased shear, despite antiplatelet therapy. This may cause activated platelet plug embolization distally, with secondary hemorrhagic transformation of the resulting ischemic infarction [7].

The incidence of DIPH in the PLUS study was within the range of the reported incidence of DIPH from $0 \%$ to $10 \%$ [3, $13,14,18-22]$ Sweid et al. reported that $55 \%$ of DIPHs occurred within the first week and that the use of $>1$ PEDs and the baseline P2Y12 value were predictors of DIPH [13]. Cruz et al. reported that DIPH occurred within the ipsilateral cerebral hemisphere, which is anatomically remote from the treated aneurysms [21]. The IntrePED study reported an IPH rate of $2.4 \%$, which did not vary by aneurysm size or location [5]. In the PLUS cohort, all DIPH events occurred in the early postoperative period, and a location in the distal anterior circulation was an independent predictor of DIPH. PED use may cause hemodynamic alterations or the formation of microemboli, causing DIPH in distal anterior circulation aneurysms; in fact, the flow and wall shear stress are higher in the ipsilateral middle cerebral artery than in the contralateral middle cerebral artery in patients with hemorrhage after PED placement [23].

\section{Predictors of Neurological Compression Symptoms and Mortality}

In the PLUS study, the rate of cranial neuropathy or brainstem symptoms caused by aneurysm compression was higher in the early postoperative period than during later follow-up, and adjunctive coiling was an independent predictor of neurological compression symptoms. In the IntrePED study, adjunctive coiling resulted in a higher neurological morbidity rate than PED treatment alone $(12.5 \%$ vs $7.8 \% ; p=0.13)$ [24]. Siddiqui et al. described a patient who experienced acute thrombosis of the PED after the procedure, which was likely related to the mass effect and thrombogenicity of the dense coil mass [25]. Together, these data suggest that adjunctive coiling can increase or aggravate the compression effect by transforming a soft, pulsating mass into a hard volume that occludes the aneurysm while shifting the pulsation of the parent artery to the surrounding tissue to enhance or provoke compression symptoms [26]. Compared to the above studies, the PLUS study analyzed cranial neuropathy or brainstem symptoms caused by aneurysm compression due to the PED after the procedure.

Previously published studies have reported mortality rates ranging from 0 to $7 \%$ during the early postoperative period; similarly, the mortality rate in the PLUS cohort was within this range, with $>80 \%$ of cases of mortality occurring in the early postoperative period [14, 18, 27, 28]. In the IntrePED study, the rate of neurologic mortality was $3.8 \%$, highest in the posterior circulation group (10.9\%) and lowest in the anterior circulation ICA $<10 \mathrm{~mm}$ group (1.4\%) [5]. The risk factors for neurologic mortality have not been reported; however, the PLUS study identified an aneurysm size $>10 \mathrm{~mm}$ and basilar artery aneurysms as independent predictors of mortality. Most of the cases of mortality in the PLUS cohort were related to postoperative DAR/DIPH or major ischemic stroke, which, in turn, were independently predicted by the aneurysm size and location in the basilar artery.

\section{Functional Outcomes}

In the IntrePED study, PED treatment with coiling required a significantly longer procedure and resulted in higher neurological morbidity than PED treatment alone ( $12.5 \%$ vs. $7.8 \%$, respectively). However, Lin et al. reported that the rates of neurological complications and favorable outcomes were similar for PED plus coiling and PED alone (complications: $10.3 \%$ vs $8.0 \%$; mRS score $0-2: 93.1 \%$ vs $94.7 \%$, respectively) [29]. Sweid et al. reported that $92.4 \%$ of patients achieved a good functional outcome (mRS score $<2$ ) with flow diverters and increasing age (especially ages $>75$ years); 
however, a large aneurysm size, posterior circulation aneurysms, and acute SAH were independent predictors of an unfavorable outcome [13]. Posterior circulation aneurysms are associated with lower odds of good functional outcomes because impairment of the critical functions of the brainstem causes severe effects compared to the effects of aneurysms in the anterior circulation. The rate of good functional outcomes in the PLUS study is similar to those reported by Sweid et al. [13]. and Beydoun et al. [30]. Moreover, adjunctive coiling can increase or aggravate compression symptoms, affecting functional outcomes.

Overall, the PLUS study showed that the risk factors related to ischemic stroke complications of PED treatment differ between the early postoperative period and later follow-up periods, with a greater likelihood of ischemic stroke in the basilar artery during the early postoperative period than in the distal anterior circulation during later follow-up. Additionally, we identified previously unreported independent predictors of ischemic stroke, which include successful adjustment or unsuccessful device deployment and the degree of postoperative occlusion immediately following the procedure. Furthermore, predictors of DAR, DIPH, mortality, and neurological compression symptoms identified in the PLUS cohort in a Chinese population are similar to those identified in large studies in Western populations [3, 6, 7]. Finally, the PLUS study provides predictive factors to assess the risk of complications in Chinese patients with intracranial aneurysms treated with a PED.

\section{Limitations}

The limitations of this study include the biases inherent to the retrospective design of this study and variations in PED treatment and management among different centers.

\section{Conclusion}

The PLUS study demonstrated low rates of complications and mortality in patients with intracranial aneurysms treated with the PED in China. Moreover, the PLUS study identified novel independent predictors of ischemic stroke complications related to PED treatment, including success with adjustment or unsuccessful device deployment and the degree of postoperative occlusion immediately after the procedure. Additionally, this study identified that the risk factors for PED-related ischemic stroke complications differed between the early postoperative period and later follow-up. Together, these factors can predict the risk of complications related to PED use in Chinese patients with intracranial aneurysms.

Supplementary Information The online version contains supplementary material available at https://doi.org/10.1007/s13311-020-00990-8.
Acknowledgments The authors acknowledge Medtronic for editorial assistance.

Funding This study was sponsored by the National Key Research and Development Plan of China (grant number: 2016YFC1300800), the National Natural Science Foundation of China (grant numbers: 81220108007, 81801156, 81801158, 81471167, and 81671139), the Special Research Project for Capital Health Development (grant number: 2018-4-1077), and Medtronic, Inc.

\section{Compliance with Ethical Standards}

Conflict of Interest The authors declare that they have no conflict of interest.

\section{References}

1. Becske T, Brinjikji W, Potts MB, et al. Long-term clinical and angiographic outcomes following pipeline embolization device treatment of complex internal carotid artery aneurysms: Five-year results of the pipeline for uncoilable or failed aneurysms trial. Neurosurgery. 2017; 80:40-48

2. Hanel RA, Kallmes DF, Lopes DK, et al. Prospective study on embolization of intracranial aneurysms with the pipeline device: The premier study 1 year results. J Neurointerv Surg. 2020; 12 : $62-66$

3. Kallmes DF, Brinjikji W, Boccardi E, et al. Aneurysm study of pipeline in an observational registry (aspire). Interventional neurology. 2016; 5:89-99

4. Nelson PK, Lylyk P, Szikora I, Wetzel SG, Wanke I, Fiorella D. The pipeline embolization device for the intracranial treatment of aneurysms trial. AJNR. American journal of neuroradiology. 2011; $32: 34-40$

5. Kallmes DF, Hanel R, Lopes D, et al. International retrospective study of the pipeline embolization device: A multicentre aneurysm treatment study. AJNR. American journal of neuroradiology. 2015; 36:108-115

6. Brinjikji W, Lanzino G, Cloft HJ, et al. Risk factors for ischaemic complications following pipeline embolization device treatment of intracranial aneurysms: Results from the intreped study. AJNR. American journal of neuroradiology. 2016; 37:1673-1678

7. Brinjikji W, Lanzino G, Cloft HJ, Siddiqui AH, Kallmes DF. Risk factors for haemorrhagic complications following pipeline embolization device treatment of intracranial aneurysms: Results from the international retrospective study of the pipeline embolization device. AJNR. American journal of neuroradiology. 2015; 36:23082313

8. Kim JS, Lee HB, Kwon HS. Rnf213 polymorphism in intracranial artery dissection. J Stroke. 2018; 20:404-406

9. Ni J, Han F, Yuan J, et al. The discrepancy of neurological diseases between China and western countries in recent two decades. Chin Med J (Engl). 2018; 131:886-891

10. Colby GP, Bender MT, Lin LM, et al. Declining complication rates with flow diversion of anterior circulation aneurysms after introduction of the pipeline flex: Analysis of a single-institution series of 568 cases. Journal of neurosurgery. 2018; 129:1475-1481

11. Altman DG. Practical statistics for medical research. Taylor \& Francis; 1990

12. Kallmes DF, Brinjikji W, Cekirge S, et al. Safety and efficacy of the pipeline embolization device for treatment of intracranial aneurysms: A pooled analysis of 3 large studies. Journal of neurosurgery. 2017; 127:775-780 
13. Sweid A, Starke RM, Herial N, et al. Predictors of complications, functional outcome, and morbidity in a large cohort treated with flow diversion. Neurosurgery. 2019

14. Brinjikji W, Murad MH, Lanzino G, Cloft HJ, Kallmes DF. Endovascular treatment of intracranial aneurysms with flow diverters: A meta-analysis. Stroke. 2013; 44:442-447

15. Gory B, Berge J, Bonafe A, et al. Flow diverters for intracranial aneurysms: The diversion national prospective cohort study. Stroke. 2019; 50:3471-3480

16. Rouchaud A, Brinjikji W, Lanzino G, Cloft HJ, Kadirvel R, Kallmes DF. Delayed haemorrhagic complications after flow diversion for intracranial aneurysms: A literature overview. Neuroradiology. 2016; 58:171-177

17. Cebral JR, Mut F, Raschi M, et al. Aneurysm rupture following treatment with flow-diverting stents: Computational haemodynamics analysis of treatment. AJNR. American journal of neuroradiology. 2011; 32:27-33

18. Berge J, Biondi A, Machi P, et al. Flow-diverter silk stent for the treatment of intracranial aneurysms: 1-year follow-up in a multicentre study. AJNR. American journal of neuroradiology. 2012; 33:1150-1155

19. Brinjikji W, Kallmes DF, Cloft HJ, Lanzino G. Age-related outcomes following intracranial aneurysm treatment with the pipeline embolization device: A subgroup analysis of the intreped registry. Journal of neurosurgery. 2016; 124:1726-1730

20. Byrne JV, Beltechi R, Yarnold JA, Birks J, Kamran M. Early experience in the treatment of intra-cranial aneurysms by endovascular flow diversion: A multicentre prospective study. PLoS One. 2010; 5

21. Cruz JP, Chow M, O'Kelly C, et al. Delayed ipsilateral parenchymal haemorrhage following flow diversion for the treatment of anterior circulation aneurysms. AJNR. American journal of neuroradiology. 2012; 33:603-608

22. McAuliffe W, Wycoco V, Rice H, Phatouros C, Singh TJ, Wenderoth J. Immediate and midterm results following treatment of unruptured intracranial aneurysms with the pipeline embolization device. AJNR. American journal of neuroradiology. 2012; 33: $164-170$
23. Shakur SF, Aletich VA, Amin-Hanjani S, Hussein AE, Charbel FT, Alaraj A. Quantitative assessment of parent vessel and distal intracranial haemodynamics following pipeline flow diversion. Interventional neuroradiology: journal of peritherapeutic neuroradiology, surgical procedures and related neurosciences. 2017; 23:3440

24. Park MS, Kilburg C, Taussky P, et al. Pipeline embolization device with or without adjunctive coil embolization: Analysis of complications from the intreped registry. AJNR. American journal of neuroradiology. 2016; 37:1127-1131

25. Siddiqui AH, Kan P, Abla AA, Hopkins LN, Levy EI. Complications after treatment with pipeline embolization for giant distal intracranial aneurysms with or without coil embolization. Neurosurgery. 2012; 71:E509-513; discussion E513

26. Szikora I, Marosfoi M, Salomváry B, Berentei Z, Gubucz I. Resolution of mass effect and compression symptoms following endoluminal flow diversion for the treatment of intracranial aneurysms. AJNR. American journal of neuroradiology. 2013; 34:935939

27. Arrese I, Sarabia R, Pintado R, Delgado-Rodriguez M. Flowdiverter devices for intracranial aneurysms: Systematic review and meta-analysis. Neurosurgery. 2013; 73:193-199; discussion 199-200

28. Velioglu M, Kizilkilic O, Selcuk H, et al. Early and midterm results of complex cerebral aneurysms treated with silk stent. Neuroradiology. 2012; 54:1355-1365

29. Lin N, Brouillard AM, Krishna C, et al. Use of coils in conjunction with the pipeline embolization device for treatment of intracranial aneurysms. Neurosurgery. 2015; 76:142-149

30. Beydoun HA, Azarbaijani Y, Cheng H, Anderson-Smits C, Marinac-Dabic D. Predicting successful treatment of intracranial aneurysms with the pipeline embolization device through metaregression. World neurosurgery. 2018; 114:e938-e958

Publisher's Note Springer Nature remains neutral with regard to jurisdictional claims in published maps and institutional affiliations. 